NASA Technical Memorandum 102433

AIAA-90-0354

\title{
Vapor Condensation on Liquid Surface Due to Laminar Jet-Induced Mixing: The Effects of System Parameters
}

Chin-Shun Lin

Analex Corporation

NASA Lewis Research Center

Cleveland, Ohio

and

Mohammad M. Hasan

Lewis Research Center

Cleveland, Ohio

Prepared for the

28th Aerospace Sciences Meeting

sponsored by the American Institute of Aeronautics and Astronautics Reno, Nevada, January 8-11, 1990

\section{N/SN}

(NASA-TM-102433) VAPOR CONDENSATION ON

N90-13751

LIQUIO SURFACF DUF TU LAMINAR JET-INDUCED

MIXING: THE EFFECTS OF SYSTEM PARAMETERS

(NASA) $21 \mathrm{P}$

CSCL 200

Unc135
$63 / 34 \quad 0252617$ 







VAPOR CONDENSATION ON LIQUID SURFACE DUE TO LAMINAR JET-INDUCED MIXING:

\title{
THE EFFECTS OF SYSTEM PARAMETERS
}

\author{
Chin-Shun Lin* \\ Analex Corporation \\ NASA Lewis Research Center \\ Cleveland, Ohio 44135 \\ and \\ Mohammad M. Hasan* \\ National Aeronautics and Space Administration \\ Lewis Research Center \\ Cleveland, Ohio 44135
}

\section{SUMMARY}

The effects of system parameters on the interface condensation rate in a laminar jet-induced mixing tank are numerically studied. The physical system consists of a partially-filled cylindrical tank with a slightly-subcooled jet discharged from the center of the tank bottom toward the liquid-vapor interface which is at a saturation temperature corresponding to the constant tank pressure. Liquid is also withdrawn from the outer part of the tank bottom to maintain the constant liquid level. The jet velocity is selected to be low enough such that the free surface is approximately flat. The effect of vapor superheat is assumed to be negligible. Therefore, the interface condensation rate can be determined from the resulting temperature field in the liquid region alone. The nondimensional form of the steady-state conservation equations are solved by a finite-difference method for various system parameters including liquid height to tank diameter ratio, tank to jet diameter ratio, liquid inflow to outflow area ratio, and a heat leak parameter which characterizes the uniform wall heat-flux. Detalled analyses based on the numerical solutions are performed and simplified equations are suggested for the prediction of interface condensation rate.

\section{NOMENCLATURE}

A surface area of central jet, outflow, or interface

B tank to jet diameter ratio, $D / d$

$C_{p}$ specific heat at constant pressure

D tank diameter

d jet diameter

g gravitational acceleration

* Member, AIAA. 
H liquid height from the tank bottom

$h_{c}$ condensation heat-transfer coefficient

$h_{f g}$ latent heat of condensation

Ja Jakob number, $C_{p}\left(T_{s}-T_{j}\right) / h_{f g}$

$k$ thermal conductivity

$m_{c}$ condensation mass flux

Nh wall heat-flux parameter, $N h=q_{w} D / k\left(T_{s}-T_{j}\right)$

p pressure

$\mathrm{Pg}_{\mathrm{g}}$ equilibrium hydrostatic pressure

$p^{*}$ dimensionless pressure, $\left(p-p_{g}\right) / p u_{j}^{2}$

Q jet volume flow rate

$\mathrm{q}_{w}$ tank wall heat flux

$r$ radial coordinate measured from the centerline

$r^{*}$ dimensionless radial coordinate, $r / D$

Qe $j$ jet Reynolds number, $\rho u_{j} d / \mu$

St $c$ condensation stanton number, $h_{c} / \rho u_{j} C_{p}$

$T$ temperature

$T^{*}$ dimensionless temperature, $\left(T-T_{j}\right) /\left(T_{s}-T_{j}\right)$

u axial velocity

$u_{c}$ condensation-induced velocity (positive value)

$u^{*}$ dimensionless axial velocity, $u / u_{j}$

$u_{c}^{*}$ dimensionless condensation-induced velocity, $u_{c} / u_{j}$

$\checkmark$ radial velocity

$v^{*}$ dimensionless radial velocity, $v / u_{j}$

$v_{s}^{*}$ dimensionless radial velocity at interface

$x$ axial coordinate measured from the tank bottom

$x^{*}$ dimensionless axial coordinate, $x / 0$ 


$$
\begin{array}{ll}
x_{p} \quad \text { potential core length } \\
\mu \quad \text { dynamic viscosity } \\
\rho \quad \text { liquid density } \\
\text { - average value over the interface }
\end{array}
$$

Subscripts:

j evaluated at jet inlet

out evaluated at outflow location

s evaluated at liquid-vapor interface

c evaluated at condensation condition

$v \quad$ evaluated at vapor condition

\section{INTRODUCTION}

The pressure control of cryogenic storage tanks in space is one of the major technologies being developed in the NASA cryogenic fluids management program. The heat transferred through insulated tank walls can result in thermal stratification of the cryogenic propellant which increases the tank self-pressurization rate. A preferred method of controlling the pressure of a cryogenic storage system is the use of axial jet-induced mixing (refs. I and 2). A slightly subcooled jet can provide mixing of tank liquid and transfers heat from the interface into the bulk liquid. Vapor condensation is induced and the pressure of the tank is then reduced. Therefore, the interface condensation plays the key role in controlling the pressure of a jet-induced mixing tank.

Several studies of steady-state fluid mixing and condensation rate have been conducted. Thomas (ref. 3) measured the condensation rate of steam on water surfaces mixed by a submerged turbulent jet and found that the condensation rate was roughly proportional to the jet Reynolds number. Dominick (ref. 4) investigated the effects of jet injection angle and jet flow rate on the condensation rate in a Freon 113 tank. The interface heat transfer was observed to increase as jet injection angle became more normal to the interface. However, the average heat transfer coefficient at the interface was found to be increasing with jet Reynolds number only to a power of 0.73 . Sonin et al. (ref. 5) measured the steady-state condensation rate of steam in a water tank with condensation process dominated by the liquid-side turbulence near the interface. A correlation between the condensation rate and characteristic interface turbulent velocity was developed. Hasan and Lin (ref. 6) used a finite-difference method to solve time-averaged conservation equations along with a $k-e$ turbulence model for the prediction of turbulent velocity. The numerical prediction was in good agreement with Sonin's data except for the region close to the interface where the diffusion process was dominant. The failure of the numerical solution for the near-interface region, as stated in reference 6 , was probably due to an inappropriate turbulence model or the inappropriate turbulence boundary conditions applied at the interface. The 
effects of flow parameters, such as jet Reynolds number and Prandt 1 number on the interface condensation rate in a laminar jet-induced mixing tank was studied numerically by Lin (ref. 7). The numerical results showed that the average condensation heat-transfer coefficient increased linearly with the jet Reynolds number and varied with the liquid Prandt l number to a power of about 0.97 for $0.85 \leq \operatorname{Pr} \leq 2.65$.

Besides the flow parameters, the vapor condensation rate in a mixing tank is also dependent on the system parameters which affect directly the design of the mixing device. Lacking an adequate design data base, any overdesign of the mixing device will increase its weight and power consumption. This will lower the performance efficiency of the mixing device in reducing tank pressure. This paper presents results of a numerical investigation on the effects of system parameters on the interface condensation rate and provides useful information for the design of a mixing device.

Reference 7 showed that for a small jet to tank diameter ratio, most of the vapor condensation occurs in the central part of the interface so that the normal-gravity solution (flat interface) may be a good approximation to lowgravity condition (curved interface). Only laminar flow is considered in the present investigation. However, it is expected that the effects of system parameters on the interface condensation process will be qualitatively the same for both the laminar and the turbulent jets.

Numerical solutions of the nondimensional continuity, momentum and energy equations are obtained by a finite-difference method. Calculations cover a wide range of system parameters such as liquid height to tank diameter ratio, tank to jet diameter ratio, liquid inflow to outflow area ratio, and a parameter Nh which characterizes the uniform wall heat-flux. Detailed analyses are performed and simplified equations are suggested to predict the effects of these parameters on the condensation stanton number which characterizes the steady-state interface condensation rate.

\section{PROBLEM FORMULATION AND DESCRIPTION}

The physical system and the coordinates used to analyze the problem are shown in figure 1. A laminar axial-jet of diameter $d$ is located at the center of the bottom of a cylindrical tank of diameter D. Liquid is continuously withdrawn from the outer portion of the tank bottom to maintain a constant liquid fill level. The tank outflow to jet inflow area ratio ( $A_{\text {out }} / A_{j}$ ) is varied from 7.5 to 360 and the tank to jet diameter ratio $(0 / d)$ is varied from 12.5 to 33.3. Both the central jet and outflow velocities, $u_{j}$ and $u_{\text {out }}$ respectively, are assumed uniform. The effect of vapor superheat is neglected which implies that $C_{p v}\left(T_{v}-T_{s}\right) h_{f g} \ll 1$. Also, the effect of vapor motion on the condensation process is assumed to be negligible. The above assumptions effectively decouple the liquid region from the vapor region. Therefore, the condensation rate can be determined from the solution of the temperature field in the liquid region only.

Various fill levels are considered with the liquid height to tank diameter ratio (H/D) ranging from 0.25 to 1.25. The liquid-vapor interface is assumed to be flat (wave free), shear-free and at a constant saturation temperature, $T_{s}$. The axial jet is at a constant subcooled temperature, $T_{j}$, and the tank outflow region is assumed to have zero temperature gradient. Tank walls are 
subjected to a uniform heat leak. The dimensionless wall heat-flux parameter, Nh ( $N h \equiv q_{w} D / k\left(T_{s}-T_{j}\right)$ ), which characterizes the uniform wall heat-flux is varied from 0 to 4 . All the associated thermodynamics and transport properties are assumed constant since only small temperature variation is considered in the whole flowfield.

The energy and mass balance in the liquid region can be expressed, respectively, as

$$
\begin{gathered}
\int \rho u_{\text {out }} C_{p} T_{\text {out }} d A_{\text {out }}-\int \rho u_{j} C_{p} T_{j} d A_{j}=\int m_{c} h_{f g} d A_{s}+\int \rho u_{c} C_{p} T_{s} d A_{s}+D H q_{w} \\
\rho u_{\text {out }} A_{\text {out }}-\int m_{c} d A_{s}=\rho u_{j} A_{j}
\end{gathered}
$$

where $m_{c}, h_{f g}, c_{p}$. Tout, and $q_{w}$ are the local interfacial condensation mass flux, latent heat of condensation, specific heat at constant pressure, local outfiow temperature, and wall heat flux, respectively. The condensationinduced velocity is obtained by

$$
u_{c}=\frac{m_{C}}{\rho}
$$

By using equations (2) and ( 3 ), equation (1) becomes

$$
\int m_{c} d A_{s}=\frac{Q J a\left(\frac{T_{\text {out }}-T_{j}}{T_{s}-T_{j}}\right)-\pi D H \frac{q_{w}}{h_{f g}}}{1+J a\left(1-\frac{T_{\text {out }}-T_{j}}{T_{s}-T_{j}}\right)}
$$

where the jet volume flow rate $(Q)$, the Jakob number $(\mathrm{Ja})$, and the average outflow temperature are defined as $Q=u_{j} A_{j}, J a=C_{p}\left(T_{s}-T_{j}\right) / h_{f g}$, and $T_{\text {out }}=\int T_{\text {out }} \mathrm{dA}$ out $/ A_{\text {out }}$, respectively. The form of equation ( 4 ) is convenient to estimate the interfacial condensation mass flux by using the measurable experimental variables. The condensation mass flux at the interface is given by

$$
\begin{aligned}
\bar{m}_{c} h_{f g} A_{s} & =\int m_{c} h_{f g}{ }^{d A_{s}} \\
& =\int\left(k \frac{\partial T}{\partial x}\right)_{s} d A_{s}
\end{aligned}
$$

where $\bar{m}_{c}$ is the average condensation mass flux at the interface, and $k$ is the thermal conductivity. Thus, the condensation-induced velocity can be related to the interfacial temperature gradient by

$$
u_{c}=\frac{k\left(\frac{\partial T}{\partial x}\right)_{s}}{\rho h_{f g}}
$$


Equations (4) and (5) can be used as the termination criterion for the iterative calculation or as a check of the numerical solutions.

\section{MATHEMATICAL MODELING}

The jet-induced mixing problem considered in the present study is steadystate and incompressible with gravity acting in the vertical negative-x direction. The dimensionless forms of the governing equations, with buoyancy force resulting from the temperature gradient in the liquid neglected, are

$$
\begin{aligned}
& \frac{\partial u^{*}}{\partial x^{\star}}+\frac{\partial r^{\star} v^{\star}}{r^{\star} \partial r^{\star}}=0 \\
& \frac{\partial u^{\star}}{\partial x^{\star}}+\frac{\partial u^{\star} r^{\star} u^{\star}}{r^{\star} \partial r^{\star}}=-\frac{\partial p^{\star}}{\partial x^{\star}}+\frac{1}{B \operatorname{Re} j}\left[\frac{\partial^{2} u^{\star}}{\partial x^{\star 2}}+\frac{\partial}{r^{\star} \partial r^{\star}}\left(r^{\star} \frac{\partial u^{\star}}{\partial r^{\star}}\right)\right] \\
& \frac{\partial u^{\star} v^{\star}}{\partial x^{\star}}+\frac{\partial r^{\star} v^{\star}}{r^{\star} \partial r^{\star}}=-\frac{\partial p^{\star}}{\partial r^{\star}}-\frac{1}{B \operatorname{Re} j} \frac{v^{\star}}{r^{\star 2}}+\frac{1}{B \operatorname{Re}_{j}}\left[\frac{\partial^{2} v^{\star}}{\partial x^{*}}+\frac{\partial}{r^{\star} \partial r^{\star}}\left(r^{\star} \frac{\partial v^{\star}}{\partial r^{\star}}\right)\right] \\
& \frac{\partial u^{*} T^{\star}}{\partial x^{\star}}+\frac{\partial r^{\star} v^{\star} T^{\star}}{r^{\star} \partial r^{\star}}=\frac{1}{B \operatorname{Re}_{j} \operatorname{Pr}}\left[\frac{\partial^{2} T^{\star}}{\partial x^{\star}}+\frac{\partial}{r^{*} \partial r^{\star}}\left(r^{\star} \frac{\partial T^{*}}{\partial r^{*}}\right)\right]
\end{aligned}
$$

The nondimensional varlables in the above equations are defined as

$$
x^{*}=\frac{x}{D}, r^{*}=\frac{r}{D}, u^{*}=\frac{u}{u_{j}}, v^{*}=\frac{v}{u_{j}}, p^{*}=\frac{p-p_{g}}{\rho u_{j}^{2}}, T^{*}=\frac{T-T_{j}}{T_{s}-T_{j}}
$$

It is noted that, for convenience, the gravity term has been subtracted from the $x$-momentum equation by using the static equilibrium equation:

$$
\frac{\partial p_{g}}{\partial x}=-\rho g
$$

The relevant parameters in the governing equations are the jet Reynolds number $\left(\operatorname{Re}_{j}\right)$, the Prandt 1 number $(\operatorname{Pr})$, and tank to jet diameter ratio $(B)$ :

$$
\operatorname{Re}_{j}=\frac{\rho u_{j} d}{\mu}, \operatorname{Pr}=\frac{C_{p}{ }^{\mu}}{k}, B=\frac{D}{d}
$$

The following boundary conditions are used to solve the elliptic equations (7) to (10). At the centerline, the symmetric conditions are used:

$$
v^{\star}=0, \frac{\partial u^{\star}}{\partial r^{*}}=\frac{\partial T^{*}}{\partial r^{*}}=0
$$

Nonslip conditions are applied to the solid walls:

$$
u^{*}=v^{*}=0
$$


The bottom-wall is assumed to be adiabatic:

$$
\frac{\partial T^{\star}}{\partial x^{\star}}=0
$$

The side-wall is subjected to a uniform heat flux:

$$
\frac{\partial T^{\star}}{\partial r^{\star}}=N h
$$

where the wall heat-flux parameter $\mathrm{Nh}$ is defined by

$$
N h=\frac{q_{w} D}{k\left(T_{s}-T_{j}\right)}
$$

The interface is at saturation temperature $T_{S}$ and is assumed to be wavefree and shear-free:

$$
\frac{\partial v^{\star}}{\partial x^{\star}}=0, T^{\star}=1 \text { at } \quad x^{\star}=\frac{H}{D}
$$

The axial velocity at the interface is the condensation-induced velocity and is given, from equation ( 6$)$, by

$$
u^{*}=-u_{c}^{*}=-\frac{\operatorname{Ja}\left(\frac{\partial T^{\star}}{\partial x^{\star}}\right)_{s}}{\operatorname{Pr} B \operatorname{Re}_{j}} \text { at } x^{*}=\frac{H}{D}
$$

It should be noted that although buoyancy force is neglected, the velocity and temperature fields are still coupled through the condensation-induced velocity $u_{C}$ at the interface.

For the central jet, uniform velocity and temperature are assumed:

$$
u^{*}=1, v^{*}=0, T^{*}=0
$$

In order to maintain constant liquid level, the volume flow rate of the liquid withdrawn from the outer part of the tank bottom should be equal to the jet volume flow rate plus the condensation volume flow rate. With the assumption of uniform velocity and zero temperature gradient, the boundary conditions at the liquid-withdrawn $p l a n e$ are given by

$$
u^{*}=-\frac{A_{j}}{A_{\text {out }}}\left[1+\frac{\mathrm{Ja} \overline{\left(\frac{\partial T^{*}}{\partial x^{*}}\right)}}{\operatorname{Pr} \operatorname{Re}_{j}}\right] \text {, }
$$

and

$$
v^{*}=0, \frac{\partial T^{*}}{\partial x^{*}}=0
$$


It is evident that besides $\operatorname{Re}_{j}, \operatorname{Pr}$, and $B(=D / d)$, four additional parameters associated with the boundary conditions are:

$$
\frac{H}{D}, \frac{A_{\text {out }}}{A_{j}}, \mathrm{Ja} \text {, and } \mathrm{Nh}
$$

The average condensation heat-transfer coefficient, $\bar{h}_{C}$, and condensation Stanton number, $\overline{s t}_{c}$, which describe the interfacial heat and mass transports are defined as

$$
\begin{aligned}
& \bar{h}_{c}=\frac{\bar{m}_{c} h_{f g}}{\left\langle T_{s}-T_{j}\right\rangle} \\
& \overline{s t}_{c}=\frac{\bar{h}_{c}}{\rho u_{j} c_{p}}
\end{aligned}
$$

By using equation (4) or (5), the average condensation Stanton number can be expressed as

$$
\overline{S t}_{c}=\frac{8 \int_{0}^{0.5}\left(\frac{\partial T^{\star}}{\partial x^{\star}}\right)_{S} r^{\star} d r^{\star}}{\operatorname{Re}_{j} B \operatorname{Pr}}
$$

or

$$
\overline{S t}_{c}=\frac{Y}{R e_{j} B P r},
$$

respectively, where

$$
Y=\frac{\frac{\left(\operatorname{Pr}_{\operatorname{Re}_{j}} \overline{\mathrm{T}}_{\text {out }}^{*}\right)}{\left(\frac{D}{d}\right)}-4 \operatorname{Nh}\left(\frac{\mathrm{H}}{\mathrm{D}}\right)}{1+J a\left(1-\overline{\bar{T}}_{\text {out }}^{\star}\right)}
$$

and

$$
\bar{T}_{\text {out }}^{\star}=\frac{\bar{T}_{\text {out }}-T_{j}}{T_{s}-T_{j}}
$$


From the above analysis, the condensation Stanton number can be expressed as

$$
\overline{S t}_{c}=f\left(\operatorname{Re}, \operatorname{Pr}, \frac{D}{d}, \frac{H}{D}, \frac{A_{\text {out }}}{A_{j}}, J a, N h\right)
$$

The effect of $R_{j} j$ and $P r$ on $\overline{S t}_{c}$ has been studied in reference 7 . This paper describes the effects of the other system parameters on the interface condensation process. The calculations are conducted for jet Reynolds number (Rej) equal to 150,200, and 300 which are in laminar flow range, and for Prandt 1 number ( $P r$ ) equal to 1.25 and 2.1 which represent typical values of liquid hydrogen and nitrogen, respectively. Also, the parameters $D / d, H / D$, $A_{\text {out }} / \mathrm{A}_{\mathrm{j}}, \mathrm{Ja}$, and $\mathrm{Nh}$ are varied from 12.5 to $33.3,0.25$ to $1.25,7.5$ to 360 , 0 to 0.2 , and 0 to 4 , respectively.

\section{NUMERICAL METHOD OF SOLUTIONS}

The above elliptic partial differential equations are numerically solved by a finite-difference method. The finite-difference equations are derived by integrating the differential equations over an elementary control volume surrounding a grid node appropriate for each dependent variable (ref. 8). A staggered grid system is used such that the scalar properties, $p$ and $T$, are stored midway between the $u$ and $v$ velocity grid nodes. The bounded skew hybrid differencing (BSHD) is incorporated for the convective terms (ref. 8) and the integrated source terms are linearized. Pressures are obtained from a predictor-corrector procedure of the Pressure Implicit Split Operator (PISO) method (ref. 9) which yields the pressure change needed to acquire velocity changes to satisfy mass continuity. The governing finite-difference equations are solved iteratively by the ADI method with under relaxation until the solutions are converged.

Calculations are performed with a nonuniform grid distribution with concentration of the grid nodes in the centerline, near-wall, and near-interface regions where the gradients of flow properties are expected to be large. The nonuniform grid distribution in axial direction is generated by using an exponential function of Roberts' transformation (ref. 10) with the stretching parameter equal to 1.02 . In the radial direction, the scheme used by Cebecci and Smith (ref. II) with a constant ratio between two adjacent grid spacing is used. The 72 by 41 grid nodes which have been shown in reference 7 to give reasonable grid-independent solutions are used for all the calculations in the present study. Calculations are performed on a CRAY-XMP computer located at NASA Lewis Research Center. The convergent solutions are considered to be reached when the absolute value of (eqs. (17)-(18))/(eq. (17)) is less than 0.005 and the maximum of absolute residual sums for each dependent variables is less than $10^{-6}$.

\section{RESULTS AND ANALYSIS}

Numerical solutions showed that the flowfield near the jet region is generally independent of the parameters $\mathrm{Ja}, \mathrm{Pr}, \mathrm{Nh}$, and $\mathrm{A}_{\text {out }} / \mathrm{A}_{j}$ at least for their ranges considered in this study. The linear relation between the 
potential core length to jet diameter ratio $\left(x_{p} / d\right)$ and jet Reynolds number (Rej) obtained in reference 7, $x_{p} / d=0.0067 \mathrm{Re}_{j}$, is confirmed. The present calculations further show that the linear relation holds only when $H / d$ is greater than some critical value. As shown in figure 2, larger $R_{j} j$ and lower $\mathrm{D} / \mathrm{d}$ tend to reduce the critical value of $\mathrm{H} / \mathrm{d}$. This is because that the larger $\operatorname{Re} j$ and $d / D$ carry more jet momentum to resist the disturbance induced by the existence of free-surface.

The axial jet introduced from the bottom will turn radially outward from the centerline as it approaches the interface. Numerical solutions indicate that the radial velocity at the interface $v_{s}^{*}$ is essentially unaffected by $\operatorname{Pr}, \mathrm{Nh}$, and $A_{\text {out }} / A_{j}$. However, the $v_{s}^{*}$ is a strong function of $H / D$ and $D / d$ and is slightly affected by Ja. Figure 3 gives the distribution of radial interface velocity $\left(v_{s}^{*}\right)$ as a function of $\mathrm{Ja}, H / D$, and $D / d$. The $v_{s}^{*}$ is increasing with $\mathrm{Ja}$ and decreasing with $H / D$ and $D / d$. The peak value of $v_{S}^{*}$ is located closer to the centerline when the parameters $D / d$ and $R_{j}$ are larger, and $\mathrm{Ja}$ and $H / D$ are smaller. However, it is generally located within the region of $r^{*} \leq 0.1$. The average value of $v_{s}^{*}$ can be described by the following equation within \pm 3 percent of the exact numerical solutions:

$$
\bar{v}_{s}^{*}=0.2 \operatorname{Re}_{j}^{0.5}\left(\frac{H}{D}\right)^{-0.25}\left(\frac{D}{d}\right)^{-0.905}(1+0.226 \mathrm{Ja})
$$

for $H / d \geq 10, \mathrm{Ja} \leq 0.2,150 \leq \operatorname{Re}_{j} \leq 600, \mathrm{Nh} \leq 4$, and $1.25 \leq \operatorname{Pr} \leq 2.65$.

The average condensation Stanton number $\overline{S t}_{c}$ as a function of $A_{o u t} / A_{j}$ is shown in figure 4 for adiabatic wall condition, Nh $=0$. Calculations also include the different outflow locations for the same $A_{\text {out }} / A_{j}$. It is obvious that under adiabatic (or very low heat leak) conditions, $\overline{s t}_{c}$ is essentially independent of the liquid-withdrawn location and area. However, additional calculations for high heat leak, $N h=4$, indicate that $\overline{S t}_{c}$ is greater if the outflow location is closer to the tank wall. This is because the outflow located closer to the wall enhances the liquid circulation of the tank and convects more heat from the wall to gain a higher outflow temperature at the tank bottom. This information will be very useful in the design of a pumping system for the mixer.

The heat transfer due to condensation is carried away by the radial flow motion near the interface. Therefore, the interfacial heat transfer is expected to be greater for lower liquid fill level since the radial velocity near the interface will be greater. The variation of the average condensation Stanton number $\overline{S t}_{C}$ with $H / D$ is shown in figure 5 . For an adiabatic wall, i.e., $N h=0, \overline{S t}_{C}$ is a relatively weak function of $H / D$. However, with an increase in the wall heat-flux, the average condensation rate decreases significantly as H/D increases. As for example, figure 5 shows that for $N h=4$ and $\operatorname{Re}_{j}=150$, the condensation rate at $H / D=1.25$ is reduced by more than 60 percent compared to that at $H / D=0.25$.

Figure 6 shows the distribution of the interface temperature gradient for different values of $H / D$ and $D / d$. The interface temperature gradient at the central part of the interface, $\left(\partial T^{*} / \partial x^{*}\right)_{S}$, increases as $H / D$ decreases. 
However, $\left(\partial T^{*} / \partial x^{*}\right)_{S}$ decreases with a decrease in $H / D$ at the outer part of the interface. In contrary to the effect of $H / D$, the interface temperature gradient at the central part decreases with a decrease in $D / d$, but increases appreciably at the outer part of the interface. The average value of the interface temperature gradient is greater for smaller $0 / d$ because the larger jet diameter introduces more jet flow rate to the tank. Also seen from figure 6 , lower $H / D$ and higher $D / d$ result in the condensation being more confined in the central part of the interface. Thus, the normal-g (flat interface) solutions may be applied to the low-g condition if the liquid fill level is low and jet nozzle diameter is small enough. The effect of $\mathrm{D} / \mathrm{d}$ on $\overline{\mathrm{st}}_{\mathrm{C}}$ is given in figure 7 . For a given $H / D, \overline{S t}_{c} /(\dot{d} / D)^{2}$ approaches an asymptotic value. Thus, it can be stated that if $D / d$ is large enough, $\overline{S t}_{c}$ will be proportional to $(\mathrm{d} / D)^{2}$. For Ja $\ll 1$ and $N h \ll 1$, a correlation for predicting $\overline{s t}_{c}$ (within \pm 5 percent bound) is given by

$$
\overline{S t}_{C}=0.977\left(\frac{H}{D}\right)^{-0.025} \operatorname{Pr}^{-0.03}\left(\frac{D}{d}\right)^{-2}
$$

for $0.25 \leq H / D \leq 1.25,0.85 \leq \operatorname{Pr} \leq 2.65,150 \leq R_{j} \leq 600$, and $D / d \geq 20$. It is noted that al though $\overline{S t}_{c}$ is independent of jet Reynolds number Rej for $\mathrm{Ja} \ll<1$ and $\mathrm{Nh} \ll 1$, this may not be true for larger values of $\mathrm{Ja}$ and $\mathrm{Nh}$. From equation (21), it is evident that $D / d$ is a more important parameter affecting the interface condensation than $H / D$ and $\mathrm{Pr}$. If $H / D$ and $\mathrm{Pr}$ are close to one, $\overline{S t_{c}}=(d / D)^{2}$ may give a reasonably good prediction of interface condensation rate. In that case, $\overline{\mathrm{st}}_{c}$ is nearly a constant determined by the system geometry and the interface condensation rate can be determined by the jet volume flow rate. This conlusion also supports the finding in reference 7.

The jet subcooling is characterized by Jakob number ( $J a)$. Figure 8 shows that $\overline{\mathrm{st}}_{\mathrm{c}}$ is linearly increasing with Ja. For an adlabatic wall $(\mathrm{Nh}=0)$, the increase of $\overline{s t}_{C}$ with respect to $\mathrm{Ja}$ is very small. The effect of $\mathrm{Ja}$ on $\overline{s t}_{c}$ is enhanced at larger $\mathrm{Nh}$. However, even for high wall heat flux, $\mathrm{Nh}=4$, the difference in the value of $\overline{\mathrm{st}}_{\mathrm{c}}$ between $\mathrm{Ja}=0$ and 0.2 is still less than 4 percent. For most common cryogens such as liquid hydrogen, nitrogen, and oxygen, $\mathrm{Ja}$ is generally less than 0.2 for most practical applications. Thus, the effect of Ja can be neglected and the work of reference 7 in which $\mathrm{Ja}=0$ was assumed is also justified. It is noted that the explicit effect of $\mathrm{Ja}$ on $\overline{s t}_{c}$ is directly through the condensation-induced velocity $u_{c}^{*}$ at the interface. Thus, the negligible effect of Ja simply means that, whether $u_{c}^{*}$ at the interface is included in the calculations or not, the effect is negligible. Numerical solutions show that the maximum value of $u_{c}$, which is usually located at or near the centerline, is generally at least two orders of magnitude lower than the jet velocity. For instance, $\left(u_{c} / u_{j}\right)_{\max }$ is about 0.0077 for $\mathrm{Ja}=0.2, \mathrm{Nh}=0, \operatorname{Re}_{\mathrm{j}}=300, \mathrm{Pr}=1.25, \mathrm{H} / \mathrm{D}=1.0$, and $D / d=20$.

Heat transfer through the tank walls tends to generate thermal stratification and to increase the average temperature of the liquid. Thus, an increase in the wall heat-flux will result in the reduction of condensation rate. Figure 9 shows the linear relation between the average condensation stanton number $\overline{s t}_{c}$ and the heat leak parameter $\mathrm{Nh}$. The distribution of interface temperature gradient is shown in figure 10 . The interface temperature gradient $\left(\partial T^{*} / \partial x^{*}\right)_{s}$ is lower everywhere for $N h>0$ (wall heat leak) than that for 
$N h=0$ (adiabatic wall). It is noted that if $\mathrm{Nh}$ is large enough, say $\mathrm{Nh}=4$, a slight vaporization may occur near the tank wall region.

\section{CONCLUSIONS}

The effects of system parameters on the interface condensation rate in a laminar jet-induced mixing tank as shown in figure 1 have been numerically investigated. The selected jet velocities were low enough such that the free surface was approximately flat. The effect of vapor superheat was assumed to be negligible and the interface condensation rate was determined from the resulting temperature field in the liquid region. Solutions were obtained by solving the nondimensional form of steady-state conservation equations with a finite-difference method. Parameters investigated included liquid height to tank diameter ratio ranging from 0.25 to 1.25 , tank to jet diameter ratio ranging from 12.5 to 33.3 , liquid outflow to jet area ratio ranging from 7.5 to 360 , and side-wall heat flux parameter varied from 0 to 4 , and jet Jakob number varied from 0 to 0.2 . Under the above stated conditions, the following conclusions can be made:

- The condensation rate is essentially independent of the outflow to jet area ratio, Aout $/ A_{j}$, except for high wall heat flux.

- The average condensation Stanton number, $\overline{s t}_{c}$, linearly increases with Jakob number, Ja, and linearly decreases with the wall heat-flux parameter, Nh. The effect of Ja on $\overline{S t}_{C}$ is enhanced when $\mathrm{Nh}$ is greater than zero.

- The average condensation Stanton number, $\overline{S t}_{c}$, is decreasing with increasing liquid height to tank diameter ratio, H/D, and tank to jet diameter ratio, $D / d$. The parameters $D / d$ and $N h$ have stronger effects on $\overline{S t}_{C}$ than the parameters $H / D$ and Ja.

- For $\mathrm{Ja} \ll 1$ and $\mathrm{Nh} \ll 1, \overline{\mathrm{St}}_{\mathrm{c}}$ will be approximately equal to $(\mathrm{d} / \mathrm{D})^{2}$ if $\mathrm{D} / \mathrm{d}>20$ and $\mathrm{Pr}$ and $\mathrm{H} / \mathrm{O}$ are close to one. Thus, the interface condensation rate can be determined by the jet volume flow rate.

- Lower values of $H / D$ and $d / D$ will yield condensation more confined at the central part of the interface. Thus, normal-g (flat interface) solution may be applied to the low-g (curved interface) condition if the

liquid fill level is low and the jet nozzle diameter is relatively small.

\section{REFERENCES}

1. Aydelott, J. C., Carney, M. J., and Hochstein, J. I.: NASA Lewis Research Center Low-Gravity Fluid Management Technology Program. NASA TM-87145, 1985.

2. Poth, L. J. and Van Hook, J. R.: Control of the Thermodynamic State of Space-Stored Cryogens by Jet Mixing. J. Spacecraft and Rockets, Vol. 9, No. $5,1972$. 
3. Thomas, R. M.: Condensation of Steam on Water in Turbulent Motion. Int. J. Multiphase Flow, Vol. 5, pp. 1-15, 1979.

4. Dominick, S. M.: Mixing Induced Condensation Inside Propellant Tanks. AIAA Paper No. 84-0514, 1984.

5. Sonin, A. A., Shimko, M. A., and Chun, J. H.: Vapor Condensation onto a Turbulent Liquid - I. Steady State Condensation Rate as a Function of Liquid-Side Turbulence. Int. J. Heat Mass Transfer, Vol. 29, No. 9, pp. 1319-1332, 1986.

6. Hasan, M. M. and Lin, C. S.: Axisymmetric Confined Turbulent Jet Directed Towards the Liquid Surface From Below. AIAA Paper No. 89-0172, NASA TM 101409 , 1989.

7. Lin, C. S.: Numerical Studies of the Effects of Jet-Induced Mixing on Liquid-Vapor Interface Condensation. AIAA Paper No. 89-1744, 1989.

8. Syed, S. A., Chiappetta, L. M., and Gosman, A. D.: Error Reduction Program. NASA CR-174776, 1985.

9. Issa, R. I.: Solution of Implicitly Discretised Fluid Flow Equations by Operator-Splitting. J. Comput. Phys., Vol. 62, No. 1, 1986.

10. Roberts, G. 0.: Computational Meshes for Boundary Layer Problems. Proc. Second Int. Conf. Num. Methods Fluid Dyn., Lecture Notes in Physics, Vol. 8, Springer-Verlag, New York, pp. 171-177, 1971.

11. Cebeci, T. and Smith, A. M. O.: Analysis of Turbulent Boundary Layers. Academic, New York, 1974.î 


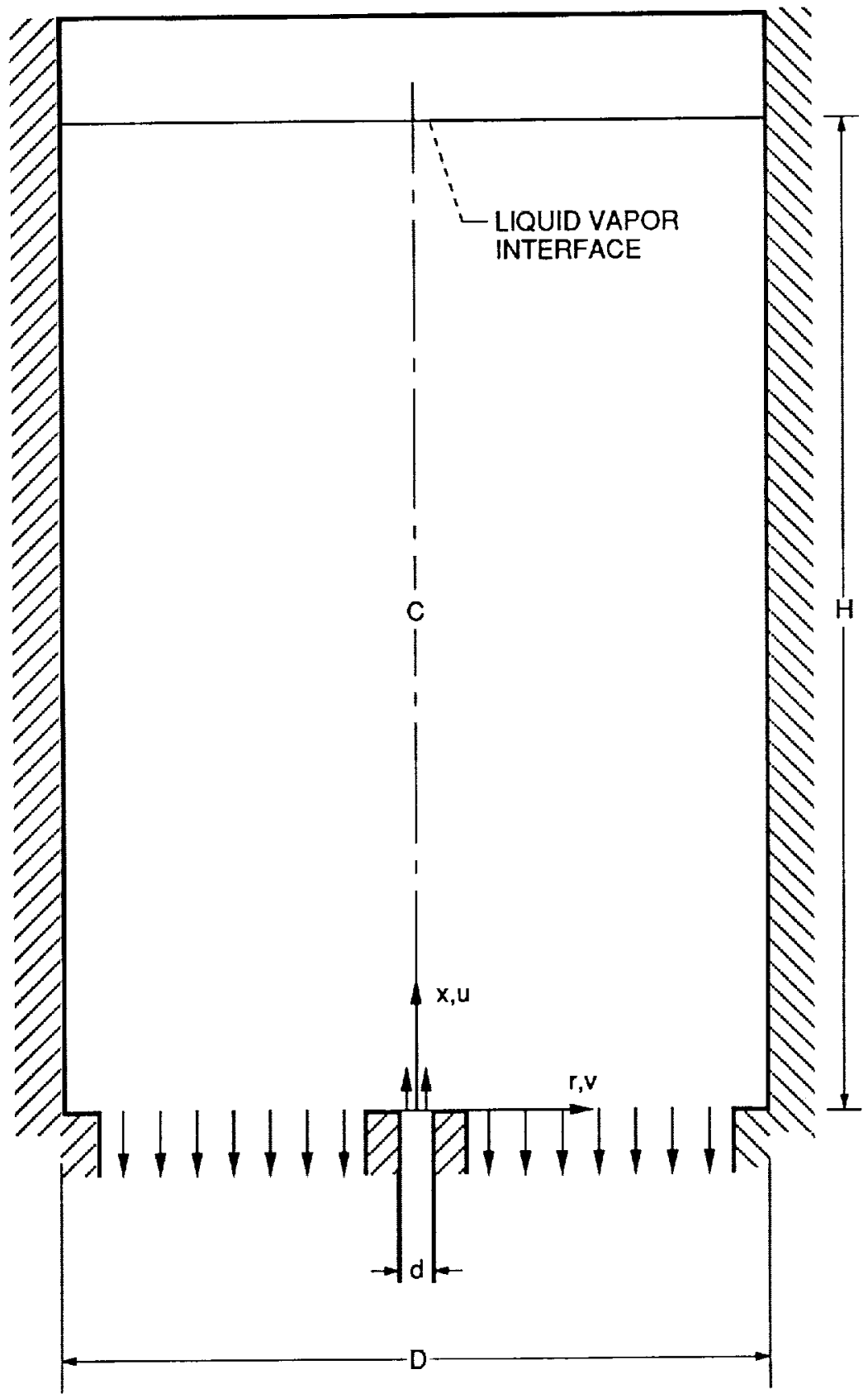

Figure 1. - Physical system and coordinates. 


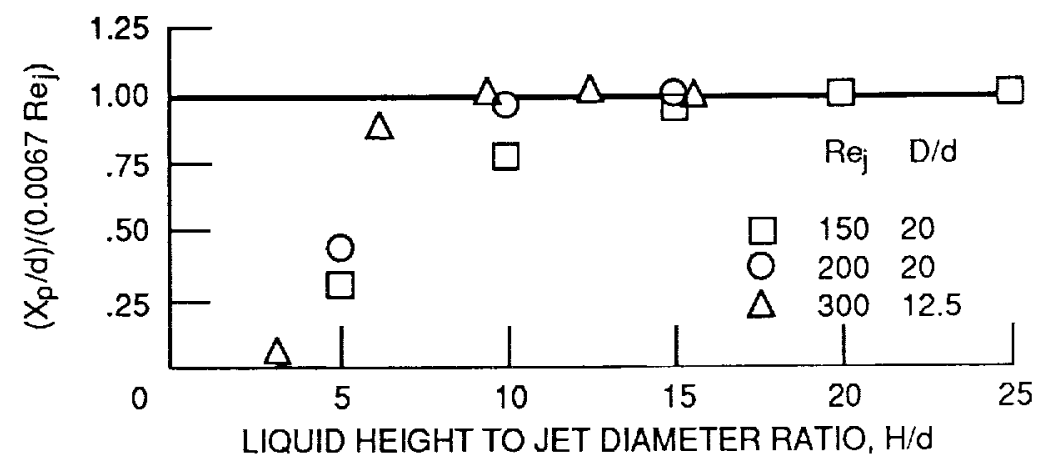

Figure 2. - Potential core length as a function of jet Reynolds number.

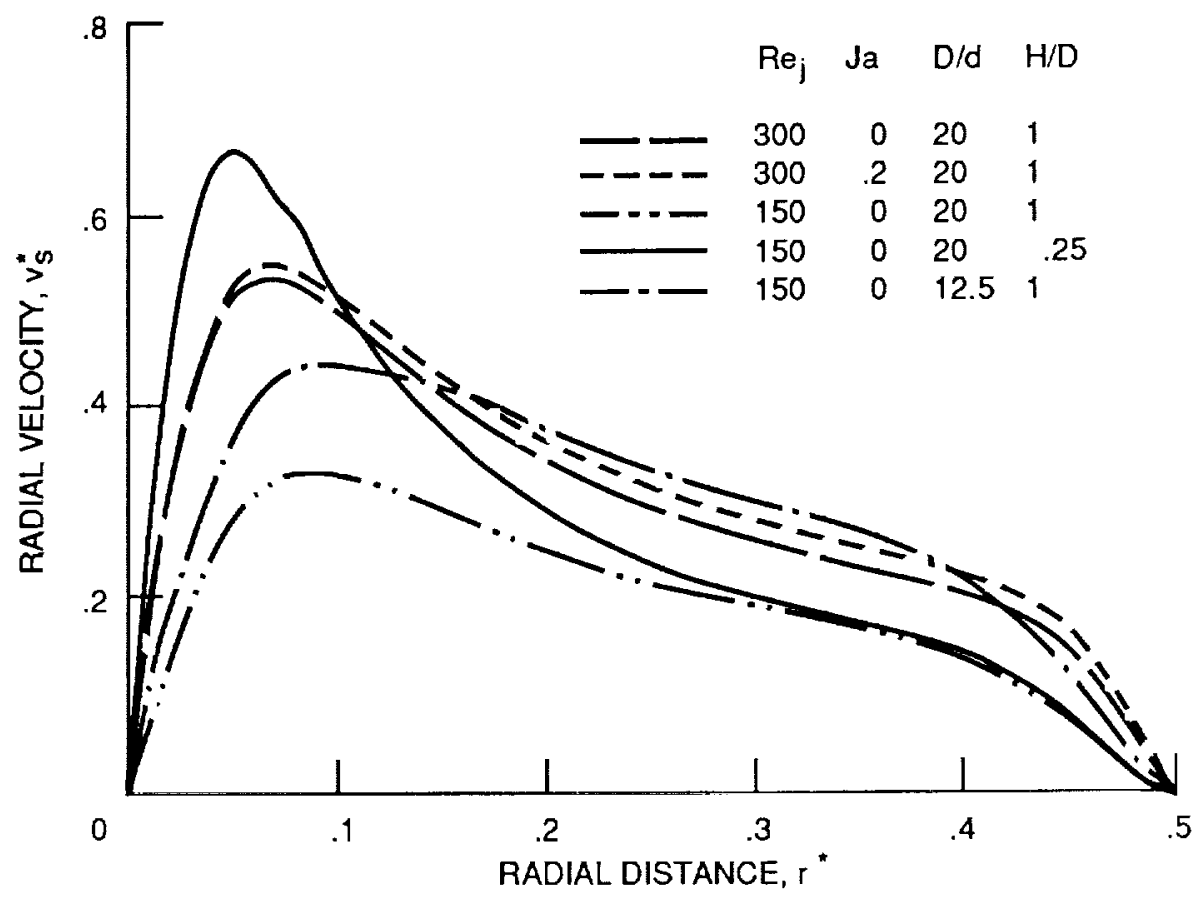

Figure 3. - Radial velocity distribution at the liquid-vapor interface for $\mathrm{Nh}=0$ and $\operatorname{Pr}=1.25$. 




Figure 4. - Condensation Stanton number as a function of outflow to jet area ratio for $\mathrm{Re},=150, \mathrm{Nh}=0$, and $\mathrm{Ja}=0$.



Figure 5. - Condensation Stanton number as a function of liquid level height for $\mathrm{Ja}=0$ and $\mathrm{A}_{\text {out }} / \mathrm{A}_{j}=357$. 


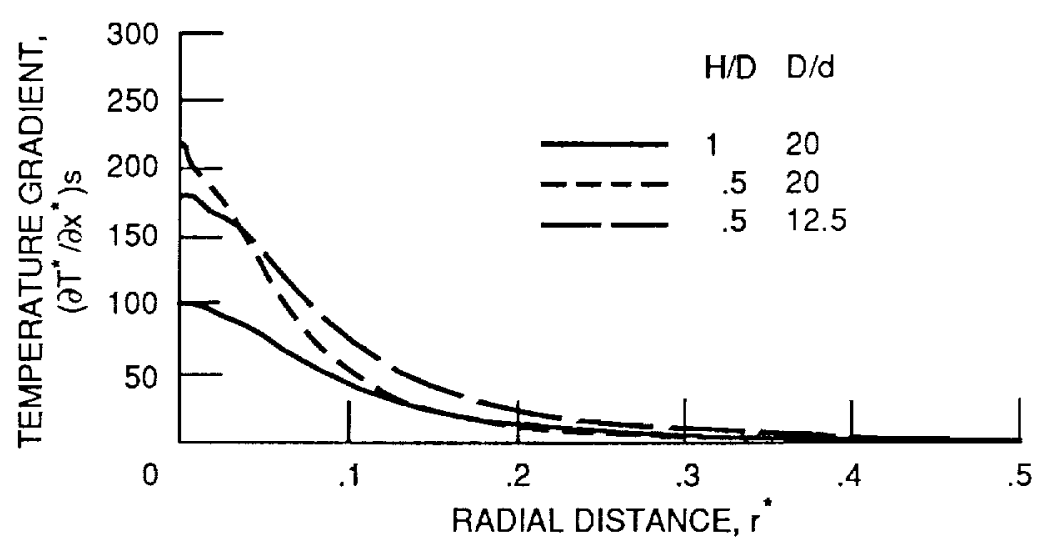

Figure 6. - Temperature gradient distribution at the interface for $\mathrm{Re}_{\mathrm{j}}=150, \operatorname{Pr}=1.25, \mathrm{Nh}=0$, and $\mathrm{Ja}=0$.

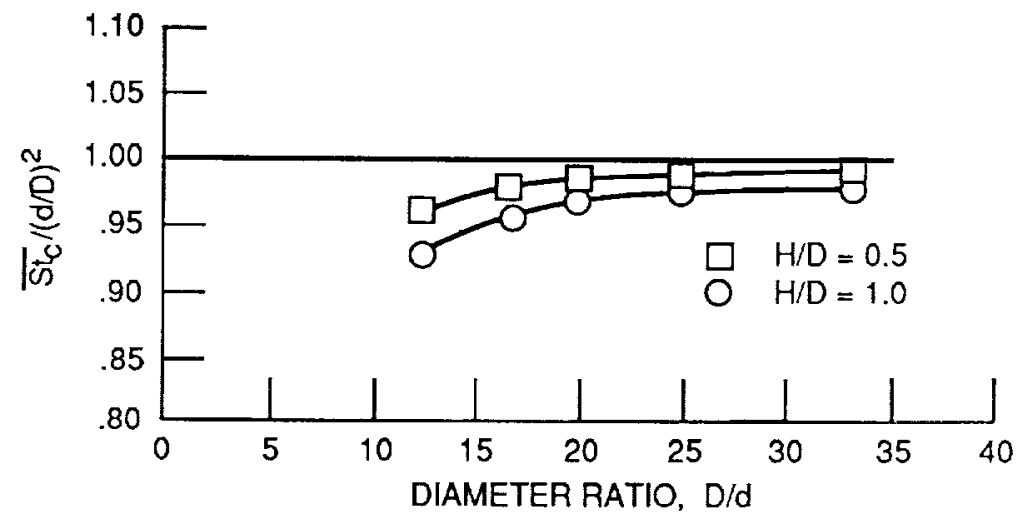

Figure 7. - Condensation Stanton number as a function of tank to jet diameter ratio for $\mathrm{Re}_{\mathrm{j}}=150, \mathrm{Pr}=1.25, \mathrm{Nh}=0$, and $\mathrm{Ja}=0$. 




Figure 8. - The effects of Jacob number and wall heat-flux parameter on the average condensation Stanton number for $\mathrm{D} / \mathrm{d}=20, \mathrm{H} / \mathrm{D}=1.0, \operatorname{Re}_{\mathrm{j}}=300$, and $\mathrm{Pr}=1.25$.

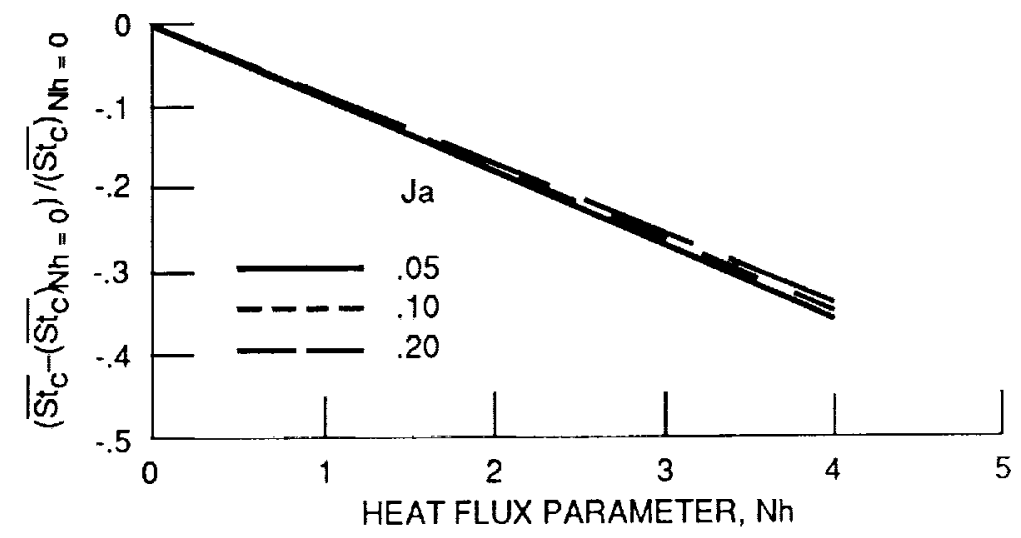

Figure 9. - The effects of wall heat-flux parameter and Jakob number on the average condensation Stanton number for $\mathrm{D} / \mathrm{d}=20, \mathrm{H} / \mathrm{D}=1.0, \operatorname{Re}_{\mathrm{j}}=300$, and $\operatorname{Pr}=1.25$. 




Figure 10. - Temperature gradient distribution at the interface for $H / D=1.0, D / d=20, A_{\text {out }} / A_{j}=357, R e_{j}=300$, and $\operatorname{Pr}=1.25$ 


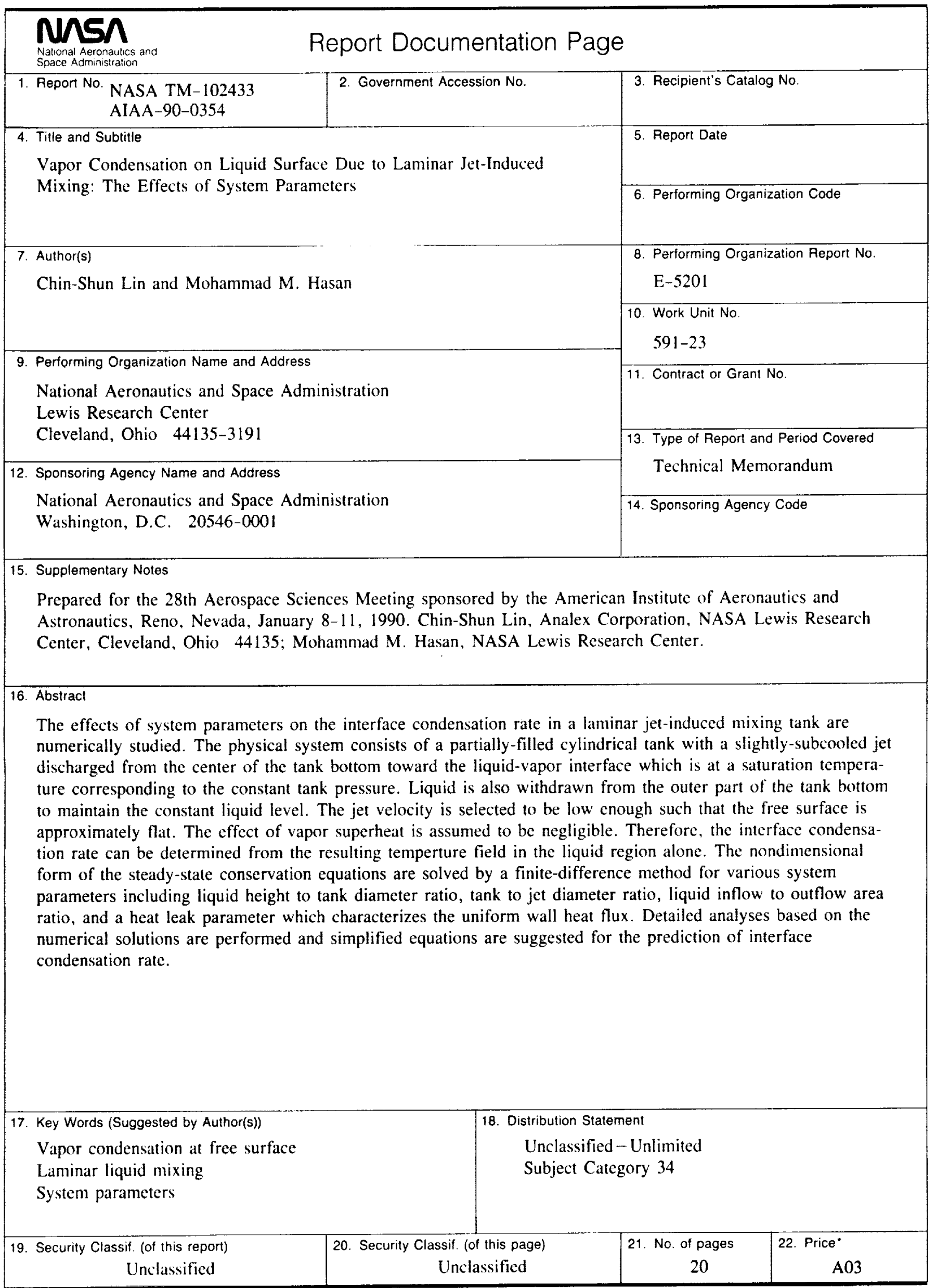

